\title{
A New Design of Test-bed For Tight-lock Coupler and Buffer
}

\author{
"Min Liu, Shengping Yang, Jie Meng, Jinghua Zhang \\ College of Mechanical and Power Engineering \\ Chongqing University of Science and Technology \\ Chongqing, China \\ *E-mail: liuyijunpp2007@163.com
}

\begin{abstract}
There is a growing concern about the safety and stability of high-speed EMUs' operation while it's running speed rises. As the significant part of the high-speed unit, the tight-lock coupler and buffer are demanded by various requirements. To ensure the quality and property, comprehensive experiments of different parts of tight-lock coupler are imperative. This article focuses on the structure of equipment, principle of operation and measurement of test-bed for testing the performance aspects of overhauled tight-lock coupler and buffer. The test-bed could be used to do the connection, de-coupling experiments of coupler in different conditions and to check the property of coupler and buffer. Furthermore, with the intelligent control, survey and management of computer, the related experimental data could be synchronized displayed and analyzed in the LCD-panel of the console. It has been confirmed that the test-bed could meet the requirement of field test after successfully used in Chongqing rail transit line 3 in recent years.
\end{abstract}

Key words-tight-lock coupler; buffer; test-bed; performance test

\section{INTRODUCTION}

With the rapid development of rail transit, the high-speed Multiple Units (EMUs) have been widely used in our country. In the course of running speed raising, there is a growing concern about the safety and stability of high-speed EMUs' operation. As the significant parts of the high-speed EMUs, the tight-lock coupler and buffer are demanded various requirements [1]:
1) The gap of the EMUs coupler's connection should be narrow to the greatest extent.

2) In order to make the fast implement of mechanical, air and electronic connection, the EMUs coupler should be easily connected and de-coupled.

3) Greater buffer capacity of coupling buffer is required to reduce the longitudinal impact of the proper operation and coupling.

4) There should be an energy absorption device to effectively protect the EMUs and passengers when the high-speed train was beyond the permissive couple speed. (eg. The train frontal crash accident).

Because the tight-lock coupler could satisfy the requirements above, it is widespread used in multiple units and urban rail trains. To guarantee the property and quality, comprehensive experiments of different parts are imperatively, including the performance tests of connection and de-coupling of the coupler and the buffer device when the coupler connected straightly in an alignment state, the load experiment of tensile strength, the gas leak test, the performance tests of connection and de-coupling of the coupler and the buffer device when there is a left and right offset or upper and lower offset between the two units [2].

According to the technical requirement described above, the test-bed for tight-lock coupler the coupling test-bed that is able to simulate the working reality. In the stage No.1 of Chongqing rail transit line 3 construction, our test-bed of tight-lock coupler and buffer could be used to check the property of coupler and buffer, to do the connection and de-coupling experiments of coupler in different conditions, and with the intelligent control, survey and management of computer, the related experimental data could be 
synchronized displayed and analyzed in the LCD-panel of the console.

\section{The COMPONENT OF TEST-BED FOR TIGHT-LOCK COUPLER AND BUFFER}

The general layout of the test-bed of coupler and buffer is horizontal (Fig1 and Fig2), which could fully simulated the actual operational condition to make the experiment accurate and reliable [3].

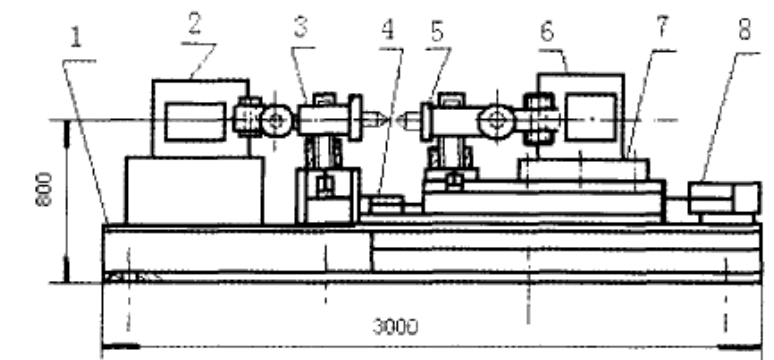

1-machine frame 2-buffer

3- fixed coupler 4- hydraulic system

5-mobile coupler 6-mobile buffer 7-mobile table 8- pressure system

Fig.1 The sketch of mechanical function of test-bed

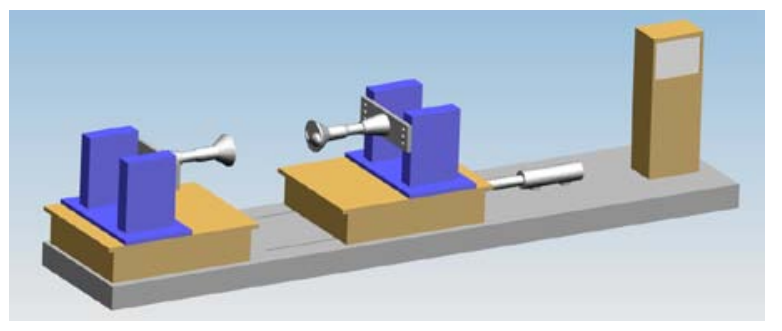

Fig.2 The sketch of test-bed for coupler and buffer

The test-bed is composed of a machine frame, a mobile table, a supporting guideway, two impact couplers, a left and right regulator system of coupler, a lifting device of coupler, a hydraulic system, a pressure system and a console.

\section{A. Machine Frame}

The machine frame is an essential part of experimental device. With the hydraulic system underneath, it is the basic framework which loads the mobile sliding table, supporting guideway, impact coupler, left and right regulator system for coupler and lifting device of coupler.
The frame welded by carbon structure steel must have sufficient strength and rigidity, it should withstand the tension load under tension experiment and the shock load when collided with the slide. There are climbing frames and handrails on both sides taking into account of ergonomics, which have reasonable structure, safe and comfortable.

\section{B. Mobile Table}

The predominant use of mobile table is detecting the performance of hooking. The major component is the dovetail guideway which made of cast iron and then finished precisely to satisfy the design require.

The tested coupler is put on the sliding table so the strength and the rigidity should be the basic requirement. Furthermore, the friction created by slide table and supporting guideway should be little.

\section{The Left and Right Regulator System for Coupler}

The left and right regulator system is designed for testing the property of coupler when there is a deflection. It is mainly composed of fixed pulleys and wire ropes which should have little friction and flexible deflection. To save work time and labor intensity, we design the hydraulic motor as the driving force.

\section{Lifting Device for Coupler}

The lifting device is designed for detecting the property of coupler when it deflected up and down. It is composed of elevator welded by carbon steel and hydro cylinder which is a standard part that can be chosen by the design parameter.

\section{E. Hydraulic System}

With hydraulic system, the power of tension test, the high pressure oil for lifting device and the left and right regulator system could be able to work. The basic components of hydraulic system are electric machine, hydraulic pump, stretching cylinder and controls. The demands of designing the hydraulic system must have stable behavior and high pressure. Meanwhile, the enough pulling force of stretching cylinder is the key point of testing the tension of coupler. 


\section{F. Pressure System}

The pressure system supports large flow of compressed gas for mobile sliding table, and air source for deconstructed test. It is composed of air compressor, gas tank, impact cylinder, air cleaning unit, muffler and controls. It is requested greater flow and less noise so the workers around would not be disturbed.

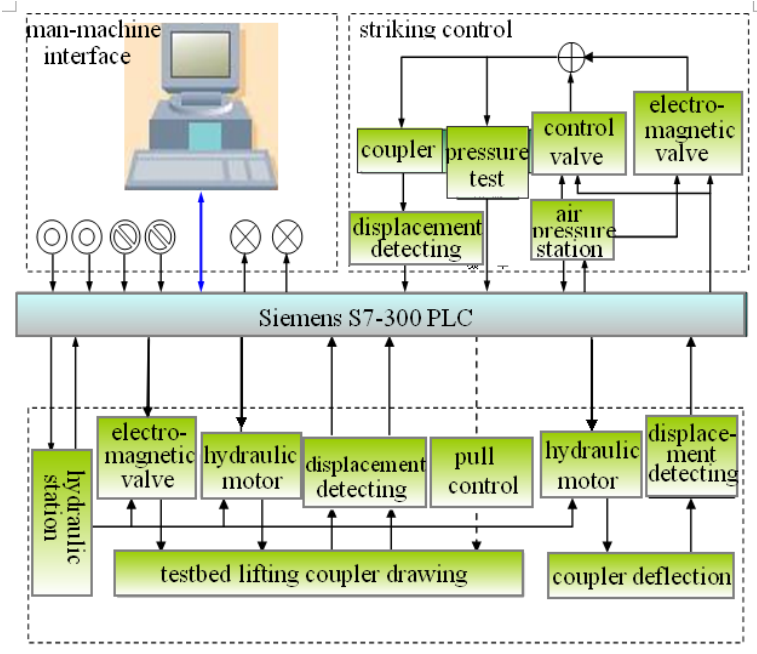

Fig.3 The control system's composition and principle

\section{G. Control system}

As Fig.3 shown, the control system is composed of test-bed, hydraulic station, air compressor station, control operation platform and sensors. The related parameter of control system could be displayed on the platform for ease to use. The artistic console is welded by carbon steel and then the controls were added.

\section{The MAIN TEChNICAL PARAMETER OF DEVICES}

1) Power supply: Three phase five wire $380 \mathrm{~V} / 50 \mathrm{~Hz}$

2) Total power: $\leq 30 \mathrm{~kW}$.

3) The power of coupling and de-coupling: hydraulic/air pressure

4) The maximum pressure of hydraulic system: 4Mpa.

5) The dimension data of test-bed: Length $\times$ Width $\times$ Height: $4900 \mathrm{~mm} \times 1500 \mathrm{~mm} \times 1500 \mathrm{~mm}$

6) The dimension data of console: $1100 \mathrm{~mm} \times 800$ $\mathrm{mm} \times 1300 \mathrm{~mm}$

\section{The MAJOR FunCtiON OF TEST-BED FOR}

\section{COUPLER AND BUFFER}

The performance measurement of this test-bed is as follows.

1) Measuring the performance of coupling, de-coupling and buffer when the coupler connected straightly in an alignment state (without deviation). And testing the load experiment of tensile strength and the gas seal when the tension is 35 tons.

2) Measuring the performance of coupling, de-coupling and buffer when there is a 30 degree offset left or right of coupling.

3) Measuring the performance of coupling, de-coupling and buffer when there is a 100 millimeters up or down.

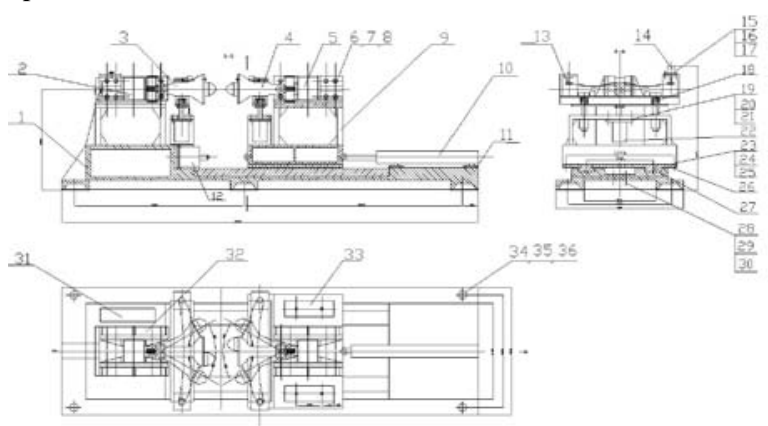

Fig.4 The assembly drawing of the mechanical drive part of the

test-bed

\section{V.The Operating PRINCIPLE OF TEST-BeD FOR COUPLER AND BUFFER}

The operating principle of test-bed for tight-lock coupler and buffer is shown in Fig.4.

A. The performance measurement of coupling, de-coupling and buffer when coupled without deviation

Step1: Through the left and right regulator system13 and lifting device22, two couplers would connect straightly without deviation. The mobile table driven by the cylinder10, moved left to dash against the coupler at the speed of 0.4-0.8irgs to test the performance of coupling and buffer. The speed of collided car is regulated by the load of driven cylinder. The performance of buffer is tested by the displacement of coupler. All the related data are shown on the panel of console. After this, the drive cylinder becomes in unloading condition [4]. 
Step2: 35 tons of pull produced by the hydraulic cylinder12 moves to right to drive the load experiment of tensile strength. The magnitude of tension is regulated by the inlet pressure of hydraulic cylinder, the gap between the two coupler is tested by displacement sensor. The related experimental data are also shown on the console panel.

Step3: The left and right regulator system13 and lifting device22 drive the coupler left and right, up and down to do the gas leak test. The gas source links the gas path system of coupler and with the pressure sensor on it, the sealing performance of gas path would be tested by the pressure.

Step4: Filling gas to the de-coupling cylinder to test the property of de-coupling.

B. Measuring the performance of coupling, de-coupling and buffer when there is a 30 degree offset left or right of coupling.

Measuring the performance of coupling, de-coupling and buffer when there is a 30 degree offset left or right of coupling which is driven by the left and right regulator system 13 . The related data would be shown in the console panel.

C. Measuring the performance of coupling, de-coupling and buffer when there is a 100 millimeters up or down.

Measuring the performance of coupling, de-coupling and buffer when there is a 100 millimeters up or down which is regulated by the lifting device22. The related data would be shown in the console panel.

\section{TESTED MEAsures}

1) Two lifting hydraulic cylinder were designed as fast and slow, the measurements of test and control are as follows [5].

a) There is a proximity switch not only to reset, but also to avoid the piston of oil cylinder drop down to the prescribed minimum.

b) The lifting of oil cylinder is tested by installing flow meter to drive the code disc in export department of hydraulic pressure unit.
2) The left and right deflected angle is controlled by two hydraulic motor which tested by the code disc.

3) The pressure of driven cylinder was regulated by the cooperation of pressure regulating valve and reversing valve. Pressure detectors are set up in air inlet to collect impact pressure value which was calculated by upper computer. With the gas tank and proportional valve, the speed of collision can be regulated by the gas flow [6].

4) The metering stroke of discharged liquid of mobile machine could calculate the displacement test of buffer.

5) With three relief valve (regulated three pressure to an certain extent), the tension hydraulic cylinder could regulate the pressure. The tension is acquired by calculation. The outlet pressure is tested by pressure sensor at oil station.

6) The tension displacement is tested by dial gauge (artificial collected data).

7) The gas leak experiment: Installing a pressure sensor in front of the de-coupling cylinder of coupler to detect the fluctuation of pressure.

8) Tommunicate PLC with upper computer which function are control, display and record [7].

\section{CONCLUSION}

The designed test-bed for tight-lock coupler and buffer in this article can effectively achieve the testing aim of coupler and buffer. With simple construction, smooth running, easy operation and lower noise, the test -bed have the function of real time parameter display, curve display, data record, analysis of historical data, experimental report printing. It plays an important role in the mechanical performance and sealing test of overhauled coupler and buffer. After successfully used in Chongqing urban transit line 3 in recent years, it has been confirmed that the test-bed could meet all the requirements of field test.

\section{ACKNOWLEDGMENT}

This paper is supported by Chongqing Research Program of Basic Research and Frontier Technology (No.cstc2013jcyjA70004), Undergraduate Education and Teaching reform project of Chongqing University of Science \& Technology (Project No.201510), and Chongqing University of Science \&Technology (No.CK2014Z27). 


\section{REFERENCES}

[1] Shao Lipeng, Yao Baotian, Dong Lisheng, "The study of the linked test bed for tight-lock coupler of high-speed EMUs," Railway vehicles, vol.50, pp.34-35, 2012.

[2] Gao Shuang, The structure and maintenance management of railway vehicles, China railway press, 2009.

[3] Wu gang. The study of test-bed for tight-lock coupler, Technology of Locomotives and Cars, vol.33, pp.34-36, 2008.

[4] Yang Jinchuan. "The test device for tight-lock coupler, Locomotives and Cars, vol.45, pp.29-31, 2009.

[5] The operation and maintenance for ChongQing light rail vehicle. Chang chun railway vehicle limited company, 2009.

[6] Zhang Zhenlin. Urban rail transit vehicle, China railway press, 2007.

[7] Li Na, "The reconstruction design of electrohydraulic control system of test bed for applies drawing ribs", The hydromatic, pneumatic and seal, vol.44, pp.45-50, 2012. 